\title{
Discrepant Advanced Directives and Code Status Orders: A Preventable Medical Error
}

\author{
Barry Meisenberg, MD*, Sohail Zaidi, MBA, MD², Lori Franks, MD¹, David Moller, PhD', David Mooradian, MD
}

'Department of Medicine, Anne Arundel Medical Center, Annapolis, Maryland; '²Department of Surgery, Anne Arundel Medical Center, Annapolis, Maryland.

re

he United States health system has been criticized for its overuse of aggressive and medically ineffective life-sustaining therapies (LST). ${ }^{1}$ Some professional societies have elevated dialog about end-of-life (EOL) care to a quality measure, ${ }^{2}$ expecting that more open discussion will achieve more "goal-concordant care" 3 and appropriate use of LST. However, even when Advanced Directives (AD) or Physician Orders for Life-Sustaining Therapy (POLST) have been created, their directions are not always followed in the hospital. This perspective discusses how preventable errors allow for use of LST even when patients designated it as unwanted. Two cases, chosen from several similar ones, are highlighted, demonstrating both human and system errors.

During the time of these events, the hospital policy required admission orders to contain a "code status" designation in the electronic medical record (EMR). All active and historical code status orders were listed chronologically and all AD and POLST documents were scanned into a special section of the EMR. Hospital policy, consistent with professional society guidelines, ${ }^{4,5}$ stated that patients with AD/POLST limiting EOL support should have individualized discussion about resuscitation options in the event of a periprocedural critical event. Automatic suspension or reinstatement of limited code orders was not permitted.

\section{CASE 1}

A 62-year-old woman with refractory heart failure was admitted with recurrence. The admitting code order was "initiate CPR/ intubation" even though a POLST order written 10 months earlier indicating "do not intubate" was visible in the EMR. A more recent POLST indicating "No CPR/No intubation" accompanied the patient in the ambulance and was placed at bedside, but not scanned. There was no documented discussion of code status that might have explained the POLST/code order disparity. Notably, during two prior admissions within the year, "full code" orders had also been placed. On the fifth hospital day, the patient was found in respiratory distress and unresponsive. A "code" was called. ICU staff, after confirming full code status, intubated the patient emergently and com-

*Corresponding Author: Barry Meisenberg MD; E-mail: BMeisenber@AAHS. org; Telephone: 443-481-5824

Published online first July 242019.

Received: April 2, 2019; Revised: May 6, 2019; Accepted: May 8, 2019

(c) 2019 Society of Hospital Medicine DOI 10.12788/jhm.3244 menced other invasive ICU interventions. Family members brought the preexisting POLST to medical attention within hours of the code but could not agree on immediate extubation. Over the next week, multiple prognosis discussions were held with the patient (when responsive) and family. Ultimately, the patient failed to improve and indicated a desire to be extubated, dying a few hours later.

\section{CASE 2}

A 94-year-old woman was admitted from assisted living with a traumatic subcapital femur fracture. Admission code orders were "initiate CPR/intubation" despite the presence in the EMR of a POLST ordering "no CPR/no intubation." The patient underwent hemiarthroplasty. There was no documented discussion of AD/POLST by the surgeon, anesthesiologist, or other operating room personnel even though the patient was alert and competent. On postoperative day one, she was found to be bradycardic and hypotensive. A code was called. After confirming full code status in the EMR, cardiac compressions were begun, followed by intubation. Immediately afterward, family members indicated that the patient had a POLST limiting EOL care. When the healthcare proxy was reached hours later, she directed the patient be extubated. The patient died 16 minutes later.

\section{DISCUSSION}

Data on the frequency of unwanted CPR/intubation due to medical error are scarce. In the US, several lawsuits arising from unwanted CPR and intubation have achieved notoriety, but registries of legal cases $^{6}$ probably underestimate the frequency of this harm. In a study of incorrect code status orders at Canadian hospitals, 35\% of 308 patients with limited care preferences had full code orders in the chart. ${ }^{7}$ It is unclear how many of these expressed preferences also had legal documents available. There was considerable variability among hospitals, suggesting that local practices and culture were important factors.

Spot audits of 121 of our own patient charts (median age 77 years) on oncology, geriatrics, and cardiac units at our institution found 36 (30\%) with AD/POLST that clearly limited life-sustaining treatments. Of these, 14 (39\%) had discrepant full code orders. A review of these discrepant orders showed no medical documentation to indicate that the discrepancy was purposeful.

A root cause analysis (RCA) of cases of unwanted resuscitation, including interviews with involved nurses, medical staff, 
TABLE. Causes of Discrepant AD/POLST and Code Status Orders with Solutions Implemented to Reduce Risk

\begin{tabular}{|c|c|}
\hline Identified Problem & Solution \\
\hline Admission process does not require code status discussion & $\begin{array}{l}\text { Admission templates to require affirmative statement that prior AD/POLST were reviewed and } \\
\text { discussion held with patient or proxy to review. }\end{array}$ \\
\hline Inadequate discussion of AD/POLST on daily interdisciplinary rounds and at shift change sign outs & Added AD/POLST discussion to standardized rounding tool and nurse and physician sign out tools. \\
\hline AD/POLST paper documents not scanned in until after discharge & $\begin{array}{l}\text { Workflow changed, so paper forms scanned in within } 24 \text { hours. POLST done in hospital to be } \\
\text { electronic, not paper for better visibility. }\end{array}$ \\
\hline $\begin{array}{l}\text { Procedural areas do not follow policy or guidelines for patients with limitations to resuscitation } \\
\text { support }\end{array}$ & $\begin{array}{l}\text { Added code status review and discussion to the procedural checklist. } \\
\text { Department and Division educational topic. }\end{array}$ \\
\hline Limited code status options & $\begin{array}{l}\text { Created new "temporary full code for procedure" code status with daily EMR reminder as long as } \\
\text { patient remains in temporary status. }\end{array}$ \\
\hline Lack of knowledge of AD/POLST legal and operational status & Department and Division educational topic. \\
\hline
\end{tabular}

and operating room, hospitalist, and medical informatics leadership, revealed several types of error, both human and system. These pitfalls are probably common to several hospitals, and the solutions developed may be helpful as well (Table).

\section{ROOT CAUSE 1: HASTE}

Haste leads to poor communication with the patient and family. Emergency departments and admitting services can be hectic. Clinicians facing time and acuity pressure may give short shrift to the essential activity of validating patient choices, regardless of whether an AD or POLST is available. Poor communication was the major factor allowing for discrepancy in the Canadian study. ${ }^{7}$ Avoiding prognostic frankness is a wellknown coping strategy for both clinicians and patients 8,9 but in all these cases, that obstacle had been overcome earlier in the clinical course of disease, leaving inattention or haste as the most likely culprit.

\section{ROOT CAUSE 2: INADEQUATE COMMUNICATION}

"It is not our hospital culture to surveille for code status discrepancies, discuss appropriateness on rounds or at sign out."

In all reviewed cases of unwanted resuscitation, numerous admitting or attending physicians failed to discuss LST meaningfully despite clinical scenarios that were associated with poor prognosis and should have provoked discussion about medical ineffectiveness. The admitting hospitalist in case 2 stated later that she had listed code choices for the patient who chose full code despite having a POLST stating otherwise. However, that discussion was not in depth, not reviewed for match to her POLST, and not documented.

Moreover, all the cases of AD/POLST and code status discrepancy were on nursing units with daily multidisciplinary rounds and where there had been twice-daily nurse-to-nurse and medical staff-to-medical staff sign out. Queries about code status appropriateness and checks for discrepant AD/ POLST and code orders were not standard work. Thus, the medical error was perpetuated.
Analysis of cases of unwanted intubation in postoperative cases indicated that contrary to guidelines, ${ }^{4,5}$ careful code status review was not part of the preoperative checklist or presurgical discussion.

\section{ROOT CAUSE 3: DECEIVED BY THE EMR}

The EMR is a well-recognized source of potential medical error. ${ }^{10,11}$ Clinicians may rely on the EMR for code status history or as a repository of relevant documents. These are important as a starting place for code status discussions, especially since patients and proxies often cannot accurately recall the existence of an AD/POLST or understand the options being presented.9,12 In case 1, clinicians partially relied upon the erroneous historical code status already in the chart from two prior admissions. This is a dangerous practice since code status choices have several options and depend upon the clinical situation. In the case of paper AD/POLST documents, the EMR is set up poorly to help the medical team find relevant documents. Furthermore, the EMR clinical decision support capabilities do not interact with paper documents, so no assistance in pointing out discrepancies is available. In addition, the scanning process itself can be problematic since scanning of paper documents was not performed until after the patient was discharged, thus hiding the most up-to-date documents from the personnel even if they had sought them. Moreover, our scanning process had been labeling documents with the date of scanning and not the date of completion, making it difficult to find the "active" order.

\section{ROOT CAUSE 4: WE DID NOT KNOW}

Interviews with different clinicians revealed widespread knowledge deficits, including appreciation of the POLST as durable across different medical institutions, effective differences between POLST and AD, location of POLST/AD within the $E M R$, recommendations of professional society guidelines on suspending DNR for procedures, hospital policy on same, the need to check for updates in bedside paper documents, and whether family members can overrule patients' stated wishes. 
Education tends to be the most common form of recommendation after RCA and may be the least efficacious in risk mitigation, ${ }^{13}$ but in this case, education reinforced by new EMR capabilities was an essential part of the solutions bundle (Table).

AD/POLST and similar tools are complex, and the choices are not binary. They are subject to change depending upon the medical context and the patient status and may be poorly understood by patients and clinicians. ${ }^{14}$ Accordingly, writing a goal-concordant code status order demands time and attention and as much nuanced medical judgment as any other medical problem faced by hospital-based clinicians. Though time-consuming, discussion with the patient or the surrogate should be considered as "standard work." To facilitate this, a mandatory affirmative statement about review of LST choices was added to admission templates, procedural areas, and clinician sign outs (Table).

Unwanted, and therefore unwarranted, resuscitation violates autonomy and creates distress, anger, and distrust among patients and families. The distress extends also to frontline clinicians who are committed to "do no harm" in every other aspect of their professional lives.

Respecting and translating patients' AD/POLST or similar tools into goal-concordant code status order is an essential professional commitment. Respect for patient safety and autonomy demands that we do it well, teach it well, and hold each other accountable.

Disclosures: The authors have nothing disclose.

\section{References}

1. Institute of Medicine. Dying in America: improving quality and honoring individual preferences near end of life Washington, DC: National Academies Pr; 2015
2. ASCO Institute for Quality: QCDR measures. http://www.instituteforquality. org/sites/instituteforquality.org/files/QOPI 2015 QCDR Measures - Narrative_0.pdf. Accessed March 3, 2019.

3. Turnbull AE, Hartog CS. Goal-concordant care in the ICU: a conceptual framework for future research. Intensive Care Med. 2017;43(12):1847-1849. https://doi.org/10.1007/s00134-017-4873-2.

4. American Society of Anesthesiology Ethics Committee. Ethical guidelines for the anesthesia care of patients with do-not-resuscitate orders or other directives that limit treatment-last amended October 2013. Accessed March $12,2019$.

5. American College of Surgeons Committee on Ethics. Statement on advanced directives by patients: "do not resuscitate" in the operating room. Bull Am Coll Surg. 2014;99(1):42-43.

6. Pope TM. Legal briefing: new penalties for disregarding advance directives and do-not-resuscitate orders. J Clin Ethics. 2017;28(1):74-81.

7. Heyland DH, Ilan R, Jiang X, You JJ, Dodek P. The prevalence of medical error related to end-of-life communication in Canadian hospitals: results of a mutlicentre observational study. BMJ Qual Saf. 2016;25:671-679. https://doi. org/10.1136/bmjas-2015-004567.

8. Robinson JD, Jagsi R. Physician-patient communication-an actionable target for reducing overly aggressive care near the end of life. JAMA Oncol. 2016;2(11):1407-1408. https://doi.org/10.1001/jamaoncol.2016.1948.

9. Ugalde A, O'Callaghan C, Byard C, et al. Does implementation matter if comprehension is lacking? A qualitative investigation into perceptions of advanced care planning in people with cancer. Support Care Cancer. 2018;26:3765-3771. https://doi.org/10.1007/s00520-018-4241-y.

10. Silversetein $\mathrm{S}$. The Syndrome of inappropriate overconfidence in computing. An invasion of medicine by the information technology industry? J Am Phys Surg. 2009; 14:49-50

11. Ratwani RM, Reider, J and Singh H. A decade of health information technology usability challenges and the path forward. JAMA. 2019;321(8):743-744. https://doi.org/10.1001/jama.2019.0161.

12. Turnbull AE, Chessare CM, Coffin RK, Needham DM. More than one in three proxies do not know their loved one's current code status: an observational study in a Maryland ICU. PLOS ONE. 2019;14(1):e0211531. https://doi.org/ https//doi.org/10.1371/journal.pone.0211531.

13. Wu AW, Lipshutz AKM, Pronovost PJ. Effectiveness and efficiency of root cause analysis in medicine. JAMA. 2008;299(6):685-687. https://doi. org/10.1001/jama.299.6.685.

14. Mirarchi F, Doshi AA, Zerkle SW, Cooney TE. TRIAD VI: how well do emergency physicians understand Physician Orders for Life-Sustaining Treatment (POLST) forms? J Patient Saf. 2015;11(1):1-8. https://doi.org/10.1097/ PTS.0000000000000165. 